\title{
Formación integral del alumnado mediante proyectos productivos multimedia en el área de agronegocios
}

The comprehensive education of students through multimedia production projects in the agribusiness area

\section{Volumen 21, Número 1 \\ Enero - Abril \\ pp. 1-25}

María Magdalena Barrera Puente

Citar este documento según modelo APA

Barrera Puente, María Magdalena. (2021). Formación integral del alumnado mediante proyectos productivos multimedia en el área de agronegocios. Revista Actualidades Investigativas en Educación, 21(1), 1-25. Doi. 10.15517/aie.v21i1.42572 


\title{
Formación integral del alumnado mediante proyectos productivos multimedia en el área de agronegocios \\ The comprehensive education of students through multimedia production projects in the agribusiness area
}

\section{María Magdalena Barrera Puente ${ }^{1}$}

\begin{abstract}
Resumen: La formación integral del alumnado en cualquier nivel educativo es un reto, es común que las intenciones queden en la planeación y lejos de la práctica. Este artículo proporciona pistas de lo que se puede realizar como complemento al currículum para formar al alumnado de manera integral, basado en la experiencia del Programa Proyectos Productivos Multimedia de Educación Continua (PPMEC). Este programa fue puesto en marcha con un grupo de alumnado de la Universidad Autónoma Agraria Antonio Narro (UAAAN) Saltillo, Coahuila, México, del año 2015 al 2019 en el área de agronegocios. El enfoque teórico se centra en las teorías y corrientes que surgieron en 1993 en la UNESCO que fundamentan el desarrollo de competencias. La metodología empleada parte de la pregunta de investigación ¿Se puede desarrollar competencias blandas y duras en el alumnado con actividades complementarias al currículum?, el enfoque que se le dio a la investigación fue de tipo mixto en una modalidad descriptiva. Por lo tanto, contempla el análisis de la experiencia de trabajo en un tiempo determinado, el diseño de la investigación es no experimental Entre las principales conclusiones destacan la evidencia de un alto grado de seguridad en sí mismos, una mayor responsabilidad en la toma de decisiones y, lo más importante, el legado de una experiencia exitosa que sirve de ejemplo e inspiración para las nuevas generaciones participantes en el programa PPMEC, se demuestra que se puede aprender a aprender, aprender a hacer y aprender a ser desde un mismo proyecto educativo.
\end{abstract}

Palabras clave: educación alternativa, competencias para la vida, aprendizaje activo

\begin{abstract}
Students' comprehensive education is a challenge in every educative level, that's why the best intentions often remain in the planning level and far from becoming a regular practice. The following article provides clues of what could be done as a complement for the curriculum to form students in an integral way, based on the experience with the PPMEC program (Productive Multimedia Projects for Continuous Education, as in its acronym in Spanish). This program was tested with a group of students from the Universidad Autonoma Agraria Antonio Narro (UAAAN) Saltillo, Coahuila. Mexico from 2015 to 2019 in the agribusiness area. The theoretical approach focusses on theories and trends emerging in 1993 at UNESCO, which are basic for development of key competences. The following article is a report of the first generation of PPMEC's experiences, logged in multiple records that form part of the outcomes of this investigation to report levels of the students' personal development, acquired knowledge, and generated value. The highlights in the main conclusions are the students' self-confidence, a bigger responsibility in decision making, and most important the legacy of a successful experience that inspirational for later generations PPMEC, proving that it's possible to learn how to learn and learn how to be, from the same educative project. People's intentions make the difference.
\end{abstract}

Keywords: alternative education, life skills, active learning

\footnotetext{
${ }^{1}$ Docente de la Universidad Autónoma Agraria Antonio Narro, Saltillo, México. MA. Administración de empresas, Universidad Autónoma del Noreste, Saltillo Coahuila, México. Doctorado en Comunicación Social de la Universidad de La Habana, La Habana, Cuba. LGAC Administración, Agronegocios, Multimedia. Orcid: $\underline{\text { https://orcid.org/0000-0003-4454-3616 }}$
}

Dirección electrónica: barrerapmagdalena@gmail.com

Artículo recibido: 26 de junio, 2020

Enviado a corrección: 10 de agosto, 2020

Aprobado: 9 de diciembre, 2020 


\section{Introducción}

El presente artículo tiene la intención de evidenciar las bases de una educación complementaria al currículum del alumnado de agronegocios de la Universidad Autónoma Agraria Antonio Narro (UAAAN), ubicada en municipio de Saltillo, Coahuila, en el norte de México. En el año 2015 dio inicio la valoración teórica y el desarrollo metodológico para transformar la estructura educativa, incorporado los pilares de la educación por competencias, fijada por la UNESCO a través de un programa que ha puesto el acento en el desarrollo y la promoción de los saberes, las actitudes participativas y, sobre todo, en el desarrollo del ser.

\subsection{Antecedentes}

La Universidad Autónoma Agraria Antonio Narro es una institución de educación superior de carácter público que fue fundada el 4 de marzo de 1923 en la localidad de la antigua Hacienda de Buenavista, en el municipio de Saltillo, Coahuila, en el norte de México, con el principal objetivo de preparar jóvenes en una disciplina profesional para las labores del campo. Debe su nombre a Antonio Narro Rodríguez, un joven saltillense que tuvo la oportunidad de estudiar agricultura en Europa y Estados Unidos donde se percató de la importancia y la diferencia que existe cuando se practica la agricultura aplicando la ciencia y la tecnología. Dos meses antes de morir, el 24 de septiembre de 1912, Antonio Narro Rodríguez había legado la parte sustancial de su fortuna personal, la Hacienda de Buenavista, con el propósito de que se creara una escuela de agricultura para captar alumnados de escasos recursos. Desde entonces, la Universidad se ha dedicado a recibir alumnado proveniente principalmente del sur del país y de Centro América, brindándoles hospedaje durante todo el semestre (Castro 2018). Es en este ambiente y con estos antecedentes es que se originó la necesidad de implementar "Proyectos Productivos Multimedia Educación Continua" que nos ocupa.

La población de La Universidad Autónoma Agraria Antonio Narro es una comunidad diversa y heterogénea. El alumnado proveniente de diferentes regiones, con características muy particulares tanto en el plano educativo como en el cultural y social que haría imposible su acceso y desarrollo en la gran mayoría de las universidades del país. En la actualidad, más del $15 \%$ de la matrícula corresponde a población indígena, más de la mitad, a población rural y el $46 \%$ del alumnado son hijos de padres con escolaridad primaria o menor. De los candidatos que presentan el examen de admisión, solo lo aprueban con calificación satisfactoria, el $6 \%$, por lo que se hace necesario apoyarlos para su nivelación con cursos 
remediales enfocados a resolver o solventar las deficiencias de formación que desarrollaron en el nivel medio superior.

En muchos casos, su lengua materna es algún dialecto y tienen acceso al español como segunda lengua. Sus conocimientos del idioma inglés son muy limitados o nulos. El tener acceso a la educación superior en una universidad agraria, para ellos, es un cambio radical en su forma de vida. Lo anteriormente expuesto justificó planear el programa PPMEC en el cual se considera promover el desarrollo de competencias en el alumnado como complemento al curriculum a la vez que se promueve la puesta en marcha de proyectos productivos en el área de agronegocios utilizando herramientas multimedia. La diversidad de experiencias acumuladas en estos años constituye una amplia aparición de procedimientos en el quehacer educativo complementario al currículum que, para algunos profesores y profesoras, podrían representar pistas creativas útiles en el intento de promover el aprendizaje significativo con miras a una formación integral.

La experiencia PPMEC parte del reconocimiento de que existen diferentes concepciones y diferentes prácticas relativas al desarrollo de competencias en el alumnado de nivel superior, y de la hipótesis de que es posible diseñar un modelo práctico a través de la intervención educativa de las personas docentes y la acción social con el alumnado, en el desarrollo de proyectos productivos. El modelo práctico de intervención puesto en marcha para la formación integral del alumnado, en la experiencia de estos cinco años, se presenta en una matriz operacional con ejes y variables que conforman una descripción clara de las acciones y procedimientos.

El objetivo general parte de analizar la experiencia de Proyectos Productivos Multimedia de Educación Continua (PPMEC) durante los años comprendidos entre los años 2015 al 2019; se pretendió atender tres objetivos específicos: el primero determinar las competencias duras y blandas desarrolladas por el alumnado participante en PPMEC. El segundo, analizar los resultados obtenidos durante la experiencia para posteriormente promover este tipo de actividades a través de la implementación de una incubadora de proyectos y puesta en marcha. Y el tercero, describir el éxito o fracaso de la puesta en marcha del proyecto PPMEC. Se parte de la pregunta de investigación: ¿Se puede desarrollar competencias en el alumnado con actividades complementarias al currículum?

A continuación se presentan los referentes teóricos en los que se fundamenta la investigación. Posteriormente, se especifican los aspectos metodológicos y los resultados. 


\section{Referentes teóricos}

\subsection{La formación integral y el desarrollo de Competencias}

La formación integral es un concepto que se ha venido perfeccionando hace ya algunos años desde la perspectiva de la Organización de las Naciones Unidas para la Educación, la Ciencia y la Cultura (UNESCO). Propone una estructura básica a partir de tres pilares: el aprender a aprender, el aprender a hacer y el aprender a ser.

Recientemente, el llamado enfoque de competencias es una interpretación de los principios de la formación integral que propone el diseño de estrategias cognitivas que promuevan los saberes con un sentido significativo para el alumnado. Es en este sentido que se ha considerado la perspectiva teórica del enfoque de competencias como el fundamento de las estrategias planeadas en el proyecto PPMEC, habilidades cognitivas que se promueven y que se encuentran implicadas en la formulación y desarrollo de proyectos productivos multimedia. Habermans (1987) propone la teoría crítica, se dedica al estudio de la filosofía en las universidades y la sociología de la educación, se enfoca a la racionalidad. La teoría que parte de la reflexión constituye la base del pensamiento crítico hacia la acción social a través de la integración y el uso del lenguaje. Es decir, un proceso de comunicación razonado. Para que se dé en un sentido significativo, las universidades deben estructurar una competencia institucional, o sea, la reproducción cultural, la integración social y la socialización. Lo objetivo corresponde a un pensamiento lógico, lo subjetivo es considerado un entendimiento lingüístico, y a la comunidad la visualiza como el racionalismo crítico que busca el aspecto argumentativo con un consenso basado en un ambiente favorable.

La teoría conceptual Raynaudo y Peralta (2017) propone que los docentes no solo debe enseñar conocimientos disciplinares, sino que también es necesario desarrollar las competencias que cada uno de del alumnado posee, no se les debe llenar solamente de conocimientos no aplicables, sino que éstos deben ser útiles para que logren tener un significado, y de esta forma se pueda aportar algo a la sociedad del conocimiento. Esta teoría considera lo afectivo más importante que lo cognitivo, el alumnado no sólo tiene que aprender conocimientos, sino que también saber relacionarse, considerar tal o cual postura de pensamiento, identificar sus emociones y decidir sobre alternativas. Las tareas del profesorado consisten en: establecer instrumentos en que el alumnado debe aprender dependiendo de la edad en la que se encuentren; elegir la mejor forma de trasmitir el conocimiento; y seleccionar la mejor manera en que estos conocimientos serán evaluados. 
Senger (1993) propone la quinta disciplina, la cual consiste en el arte y la práctica del aprendizaje colectivo. Las organizaciones utilizan prácticas colectivas de aprendizaje como centros de competencias, a través de estas prácticas se detonan los talentos y en las experiencias colectivas, se desarrollan las habilidades de las personas para aprender en conjunto. La ventaja es que las organizaciones promueven la capacidad de cada persona para generar resultados, se crea y se desarrollan las habilidades de adaptación; contempla cinco disciplinas: a) El dominio personal la organización sólo pueden aprender a través de individuos que aprenden, b) El conocimiento personal de una organización depende de un continuo aprendizaje. c) El modelo mental promueve el descubrir y retornar hacia el interior de las personas para dar significado. d) El aprendizaje en equipo permite iniciar el aprendizaje en conjunto. e) La construcción de una visión compartida, promover metas y visiones a futuro.

La persona aprende cuando logra identificarse con una identidad común, el pensamiento sistemático, permite ver la totalidad de interrelaciones en lugar de cosas aisladas, la visión compartida alienta a un compromiso de largo plazo.

La teoría del aprendizaje estratégico en la que Monereo y Pozo (1999) detallan que el personal docente es un mediador entre el alumnado y el conocimiento. De acuerdo con esta teoría, la importancia radica en el diseño de estrategias de aprendizaje que motiven al alumnado a tener interés en las actividades y así lograr un aprendizaje significativo. El aprendizaje depende de diversos factores, como son: la capacidad, la motivación, los conocimientos previos o estrategias de aprendizaje, los cuales implican tener claro el objetivo de aprendizaje, son acciones y pensamientos que se promueven durante el proceso de enseñanza aprendizaje. El alumnado tiene diferentes maneras de aprender, por lo que la persona docente tendrá la habilidad para estructurar las estrategias de aprendizaje interdisciplinarios para lograr que él construya su aprendizaje. Es a través del aprender a aprender donde se estructurarán estrategias para lograr que el alumnado construya el significado. Esta teoría se caracteriza por conectar el aprendizaje de los contenidos curriculares con el aprendizaje de los procedimientos.

Morín (2000) propone la teoría del pensamiento complejo, que se refiere a la capacidad humana de conectar las múltiples condiciones de la realidad y hacerla comprensible. Describe que cuando las personas enfrentan diversos elementos de la realidad se ven forzadas a estructurar una estrategia de pensamiento que les permita comprender lo que se les presenta. La estrategia no debe ser simple o compleja, pero sí tiene que ser reflexible y provocar el análisis. La persona posee una posibilidad infinita de interpretaciones de un mismo contexto, 
por lo que se debe considerar una retroalimentación multidisciplinaria y multi-diferenciada para lograr la construcción del pensamiento con un alto nivel de certeza. Entender la complejidad de pensar y de vivir.

Tobón (2006) propone el currículum socio-cognitivo complejo, en el cual logra identificar la relación entre la formación humana integral y las competencias. Considera al ser humano como un ser que posee determinadas características e identifica en él, una compleja naturaleza que hay que comprender y considerar para desarrollar las competencias educativas al mismo tiempo de que se promueve la formación integral.

Maldonado (2010), considera que la educación debe dar respuesta a las necesidades reales, ser pertinentes al enseñar, proporcionar al alumnado lo que requiere para lograr atender los problemas sociales. Cuando se habla del currículum, la educación se caracteriza por un núcleo de tradición, es decir, centrar la educación en los contenidos, pero el enfoque de competencias significa innovación y esos cambios son complejos, no solo en el aspecto económico, sino también de la cultura organizacional para las instituciones educativas. Los resultados se dan a través de cambiar los contenidos y la manera de enseñar, transmitir elementos pertinentes para el ser y el hacer, determinar qué necesita saber el alumnado para atender las necesidades sociales en el ámbito que le corresponde desarrollarse. Una competencia es la posibilidad de hacer bien las cosas en un determinado contexto a partir de las características, cualidades y habilidades que posea una persona. Si un sujeto puede realizar actividades articuladas con sus talentos podrá desarrollar un proceso de calidad. Es decir, será competente si articula los conocimientos con las actitudes y los valores.

Singer, Guzmán, Donoso (1999) conciben la teoría sobre las competencias blandas, surgida en los años 60, como el conjunto de habilidades no cognitivas necesarias para aprender y desempeñarse exitosamente en el trabajo, permite a una persona relacionarse con los demás, a través de los conocimientos y destrezas que se van adquiriendo durante la vida; las denominan como habilidades humanas sociales. Es importante que un profesional sea competente para un puesto, que tenga las habilidades y actitudes, pero es aún más importante que tenga una visión para desarrollar las habilidades blandas. Esto es lo que actualmente hace la diferencia. La importancia radica en ¿cómo enseñar al alumnado a poner en práctica lo que ha aprendido? además de ayudarlo a visualizar su vida profesional con un sentido de servicio colaborativo, trabajo en equipo y socialización. El Aprender a Ser revela la prevención a una deshumanización del mundo vinculada a la evolución tecnológica. 
Delors (1994) identificó que la importancia de aprender a ser significa contribuir a la formación de la esencia del hombre en su ser como individuo y socialmente en la comunidad en que le toque desarrollarse. Por el ámbito de la investigación en este marco teórico, se hace necesario considerar los términos de proyecto e incubadora de proyectos. Müller (2010) describe un proyecto como una idea planeada, que se pone en acción, con la propuesta de estrategias, en la que se contempla los medios para su realización. Está relacionada con la planeación de una idea encaminada a la producción de acciones concretas que den como resultado un elemento tangible; es decir, la producción de materiales, métodos y servicios para generar ganancias. El proyecto lo puede realizar una persona en lo individual o de forma asociativa. Una incubadora de proyectos es una herramienta para impulsar el nacimiento de una empresa, se utiliza como guía de apoyo para que las ideas de un proyecto sean factibles. Ortiz, (2018) la identifica como un proceso en el cual no basta con tener una idea, sino que es necesario diseñarla, dar congruencia y métrica, generar un plan que visualice el desarrollo y su puesta en marcha. Esta tarea no es fácil y es frecuente que los interesados se desanimen, las incubadoras de proyectos o negocios son herramientas de acompañamiento para contribuir a la conformación de nuevas empresas.

\section{Metodología}

\subsection{Enfoque}

La descripción y la interpretación de la experiencia PPMEC se realiza con base en una matriz de análisis, que incluye cinco niveles: Pedagógico, Estratégico, Organización, Multimedia e Intervención social, cada uno de ellos con sus ejes y variables. El enfoque que se le dio a la investigación fue de tipo mixto en una modalidad descriptiva, por lo tanto, contempla: el análisis de la experiencia de trabajo, y las entrevistas realizadas al alumnado involucrados en dos momentos: al inicio de la experiencia, en 2015, y al final, en 2019. Simultáneamente, se llevó el registro de la experiencia de campo, los hechos, eventos y situaciones que ocurrieron, estructurados en una línea del tiempo, de manera cualitativa y se midieron sus resultados. Se utilizaron tres instrumentos: un cuestionario para la entrevista inicial, una guía de observación, y un cuestionario para la entrevista final.

El diseño transversal de la investigación no experimental se realiza una sola vez dentro de tiempo del desarrollo de la experiencia. A través de la fuente de información, establecida por la averiguación de documentos institucionales que cuentan con información referente al objetivo de la presente investigación se fundamenta la importancia de realizar la investigación 
y la fuente de información de guías de observación y bitácoras de actividades, de donde se recabó y documentó el seguimiento a la experiencia desarrollada en un periodo 2015 al 2019.

\subsection{Unidades de análisis}

El presente trabajo se desarrolló en la Universidad Autónoma Agraria Antonio Narro ubicada en el norte de México, en el estado de Coahuila particularmente en el municipio de Saltillo, con la participación directa de $\mathbf{3 0}$ participantes 19 hombres y 11 mujeres quienes libremente se integraron al programa y que estaban inscritos en diferentes carreras y diferentes semestres de la institución: Ingeniero Agrónomo en Irrigación, Ingeniero Agrónomo en Horticultura, Ingeniero en Ciencia y Tecnología de Alimentos, Ingeniero Agrónomo Zootecnista, Licenciado en Economía Agrícola y Agronegocios, e Ingeniero Agrónomo Administrador, con la colaboración de profesores y profesoras investigadores de las mismas disciplinas quienes desarrollaron las actividades en los proyectos productivos determinados por PPMEC.

\subsection{Técnicas de recolección}

\subsubsection{Encuesta}

Con la encuesta se obtuvo información básica sobre el interés, áreas de oportunidad en la formación del alumnado que participaría en el proyecto (PPMEC) que contempla 5 variables: 1.- la decisión de participar en el proyecto, 2.- Concepción de formación educativa que proporciona la institución, 3.- conocimiento modelo educativo 4.- El área inicial en la que le gustaría trabajar y 5.- ubicación del proyecto inicial.

\subsubsection{Guía de Observación}

El segundo instrumento es una guía de observación estructurada a partir de la técnica exploratoria de acción, práctica reflexiva, que contempla las fases esenciales en un proceso: planificar, actuar, observar y reflexionar. Esta guía de observación contempla las siguientes variables: Aplicación de acciones alternativas, Planteamiento de nuevas actividades, Dinámica de la convivencia, Gestión del tiempo, Organización, Autonomía, Valores y Habilidades.

\subsection{Procesamiento de análisis}

La información recuperada se trató con un procedimiento de tipo analítico, ubicadas en categorias, se codificó y la interpretación de los resultados se realizo en un proceso de contrastar lo planificado, el actuar, observar y reflexionar apartir de las las preguntas ¿Qué? 
¿Cómo? y ¿Por qué? Se realizaron cada una de las actividades Para lo cual se elaboro la matriz de analisis que se describe a continuación.

\subsubsection{Elaboración de Matriz de Análisis}

La descripción y la interpretación de la experiencia PPMEC se realizó con base en una matriz de análisis (Figura 1), que incluye cinco niveles: Pedagógico, Estratégico, Organización, Multimedia e Intervención Social, cada uno con ejes y variables.

La revisión de literatura permitió elaborar la matriz, depurar variables e indicadores para describir la experiencia de intervención educativa en la promoción de la formación integral del alumnado en el área de los agronegocios.

El analizar el nivel Pedagógico permitió identificar la idea que tienen el alumnado del proceso educativo en el momento de ingresar al programa PPMEC. Se analiza ¿qué los motivó a elegir la carrera?, ¿cuáles son sus expectativas? y ¿qué conocimiento de su plan de estudios (currículum) consideran como significativos?; Así mismo se busca descubrir cuál es el aspecto sobre el cual se centra al acto educativo, es decir, identificar el análisis que hacen el alumnado respecto a: el aprendizaje, la acción, las vivencias, comunicación y la preparación técnica para el trabajo. Las Posibilidades de Organización trata de explicar los obstáculos y limitaciones que tienen el alumnado para organizarse, así como la respuesta pedagógica que ofrecen los profesores y profesoras para resolver la problemática de organización.

Con respecto a nivel de Contenidos se identifica los contenidos que vinculan la promoción del aprendizaje significativo a través de impulsar el saber aprender, saber hacer y saber ser. El nivel estratégico se aborda la intervención pedagógica y que son expresión del diagnóstico crítico de la realidad del alumnado en esta universidad en particular. Se analiza el rol general de la formación integral en la elaboración de proyectos productivos multimedia, es decir la posición estratégica que la formación integral debe adoptar frente a la promoción del aprendizaje significativo con sentido de vida para alcanzar los objetivos personales y profesionales. Con respecto a los objetivos sociales esta variable aborda la concepción y la prioridad que el alumnado debe identificar al vincular el conocimiento con la intervención social.

En el nivel de Organización, solo nos limitamos a describir la forma en la que los participantes se organizan para el desarrollo de las actividades. La democracia interna se analiza a partir de identificar: la libre decisión de permanecer en el proyecto con una concepción definida, la inspiración, es la esencia de vivir la experiencia los elementos clave que hacen vivir la democracia y la permanencia al pasar el tiempo. El nivel multimedia se 
describe la experiencia desarrollada, ya que en las Instituciones educativas en el área de la Agronomía la enseñanza de información y comunicación (TIC) no se consideran esenciales en la formación del alumnado, se consideran la experiencia de utilizar las TIC para el desarrollo de habilidades de comunicación escrita y oral, los resultados concretos con las actividades multimedia y aspectos generales que se pueden utilizar para el complemento curricular. El nivel de Intervención Social aborda el análisis de las acciones que se realizaron en el núcleo de formación del proyecto, donde tiene lugar el aprendizaje, la reflexión, el encuentro y el intercambio.

Figura 1. Matriz de análisis de resultados

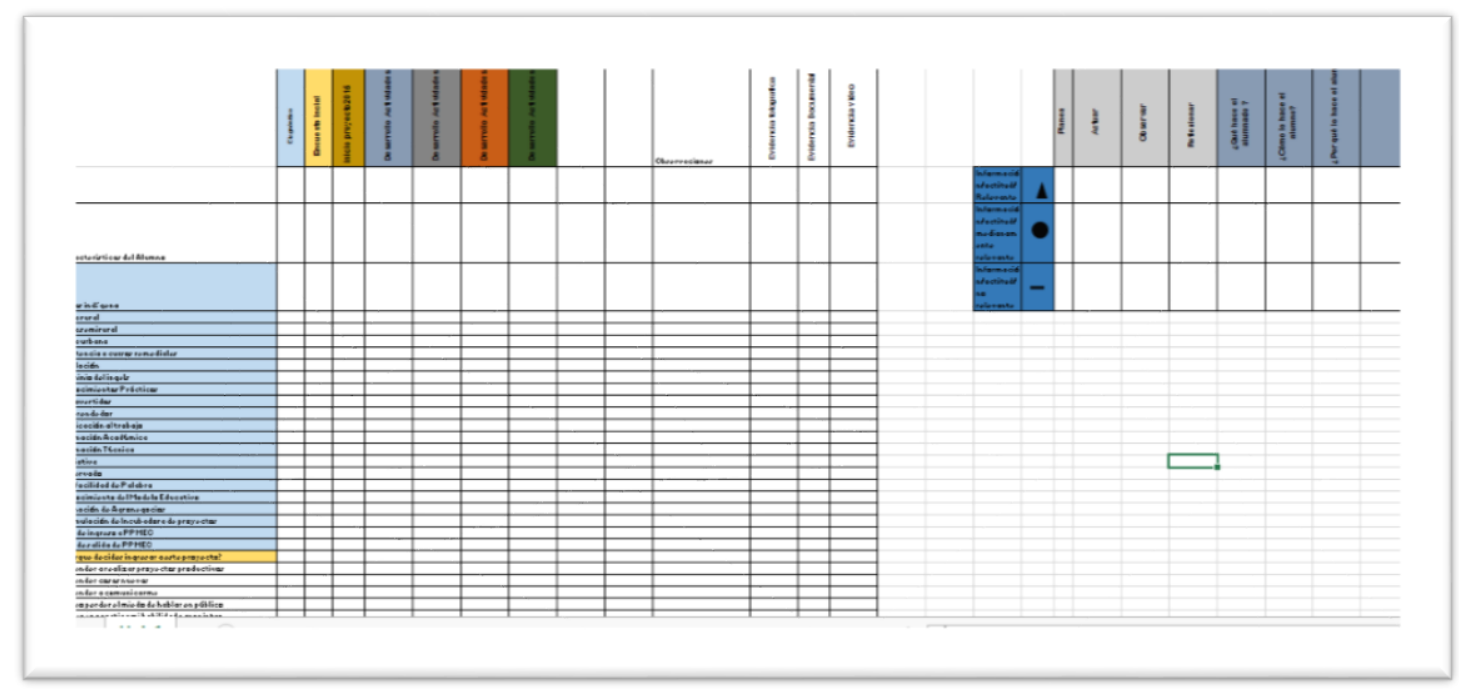

Fuente: Elaboración propia, 2015

\section{Resultados}

El proceso de implementación de PPMEC, UAAAN, fue mediante un equipo docentes, quienes desarrollaron una estrategia para introducir una innovación a un escenario educativo en un ambiente tradicional de proceso enseñanza aprendizaje.

\subsection{Nivel Pedagógico}

En la etapa inicial del diagnóstico, la Dirección General Académica de la UAAAN realizó un estudio para analizar la pertinencia de los 20 programas educativos y servicios académicos y al mismo tiempo identificar las necesidades en la formación integral del alumnado. Se realizaron entrevistas a una muestra de 466 egresados 2003-2011, 168 empleadores y a 47 expertos. Las entrevistas se realizaron entre el 15 de noviembre de 2011 y el 15 de febrero de 2012. 
Como se vio en los antecedentes, la población estudiantil de esta Universidad proviene de todas las entidades del país, de zonas indígenas, rurales, semirurales, y urbanas, por lo cual, el ambiente universitario constituye un encuentro intercultural de gran riqueza formativa, se caracteriza así por su inclusión social. En la actualidad más del 15\% de la matrícula corresponde a población indígena, más de la mitad, a población rural, el ser profesionista agrario su preparación y permanencia en la universidad está en función más del esfuerzo por aprender de ellos mismos, que de las condiciones económicas o familiares, haciendo posible la educación superior a quienes no les sería posible realizarla en la gran mayoría de las universidades del país. Del alumnado que realizan el examen de admisión, sólo el $6 \%$ obtienen una calificación satisfactoria, por lo que se hace necesario apoyar al alumnado para su nivelación con cursos remediales enfocados a resolver o solventar las deficiencias de formación que desarrollaron en el nivel medio superior. El proceso de titulación, se considera actualmente deficiente y es un problema que es común para todos los programas educativos.

El estudio reveló que el 78\% de los egresados en el periodo 2003 a 2011, estaban laborando. Las fortalezas que reconocen tener los egresados son: formación académica completa (30\%); buena actitud hacia el trabajo (13\%) y versatilidad para adaptarse (11\%). Las principales debilidades manifestadas son escaso dominio del idioma inglés, pocos conocimientos prácticos, el ser introvertidos y poco emprendedores.

La entrevista a empleadores reveló que todos los egresados cumplen con sus expectativas, otorgándoles calificaciones entre 8.42 y 9.25 en una escala del cero al diez, en la que ocho corresponden a satisfactorio. Las fortalezas de los egresados comentadas por los empleadores son: dedicación al trabajo (39\%), buena formación académica (29\%), buenos técnicos (10\%), y con iniciativa (7\%). La principal debilidad que los empleadores observan es la social, en el sentido de que son reservados y sin facilidad de palabra (UAAAN, 2012).

Con respecto a la identificación de los obstáculos para lograr una formación integral con base en el modelo educativo actual, se detectó que El Modelo Educativo "UAAAN", contempla la concepción educativa contemporánea, sobre el desarrollo de los saberes en la educación superior, enfatiza la visión integral del profesionista de la sociedad del futuro, debe conocer en forma interdisciplinaria su quehacer profesional con una formación integral del alumnado, al considerar que el currículum sea pluridimensional, es decir, que a través de este se proporcione una preparación acorde a las demandas del sector social, los capacite para el trabajo, forme el carácter y les permita ampliar el horizonte de oportunidades. Una educación global, muestra una visualización de la profesión como un todo, una formación completa, que 
le permita hacer mejor su trabajo ante las oportunidades que se le presenten, de esta manera el currículum se amplia, en el conocimiento, no la súper-especialización sino todo lo contrario, tener mayores espacios para el auto desempeño. La concepción unificadora, representa la dirección que debe observar la formación integral, mediante la unión de los procesos de investigación, de la docencia y el desempeño comunitario Modelo Educativo UAAAN (1995)

Con respecto a la Identificación de las deficiencias de formación en el área de los agronegocios, podemos mencionar que a finales de 1999 existió un programa denominado "Programa emprendedor", que tenía como objetivo despertar en el alumnado el sentido de emprender para crear. Desafortunadamente, el programa dejó de operar en el 2001 y no se ha implementado ningún programa con características similares a éste, y no existe la promoción de elaboración de proyectos encaminados a los agronegocios. El uso de las Tecnologías de Información y Comunicación es nulo la participación del alumnado en proyectos que contemplen el uso de la tecnología con fines de capacitación o servicios de asesoría hacia los productores agrícolas o médicos veterinarios.

Con base en este diagnóstico se elaboró el Programa de Proyectos Productivos Multimedia en Educación que se presentó a la Asociación Nacional de Universidades e Institutos de Educación Superior (ANUIES) en el 2015, para recibir recursos a través del Programa de Apoyo a la Formación Profesional.

La idea de PPMEC es desarrollar las competencias en el alumnado e igualmente elaborar productos multimedia que faciliten la comprensión de los temas que se ofrecen en Educación Continua, es en el año 2015 en el que el proyecto PPMEC inicia sus actividades.

El primer resultado de la encuesta inicial aplicada a los jóvenes que ingresaron a colaborar en el programa deriva en un elemento importante de análisis se detecta la concepción educativa que tiene el alumnado. Así como la decisión del porque entrar al programa, se desprende que la mayor motivación para participar fue hacer proyectos del área de agronegocios. De acuerdo con Tobón (2006): "Desde el enfoque socioformativo se identifica que las competencias propuestas en un curriculum se estructuren con la finalidad de desarrollar actividades prácticas que promuevan la formación integral y al mismo tiempo tiendan a resolver problemas reales".

A continuación se presentan los resultados del diagnóstico realizado al alumnado participante en el programa (PPMEC), en la Figura 2 se identifica la actividad principal que los motivo a intervenir en este tipo de actividad, que es: hacer proyectos en el área de los agronegocios, y por aprender a comunicarse con mayor frecuencia. 
Figura 2

Razón por la que el alumnado decidió participar en el proyecto PPMEC

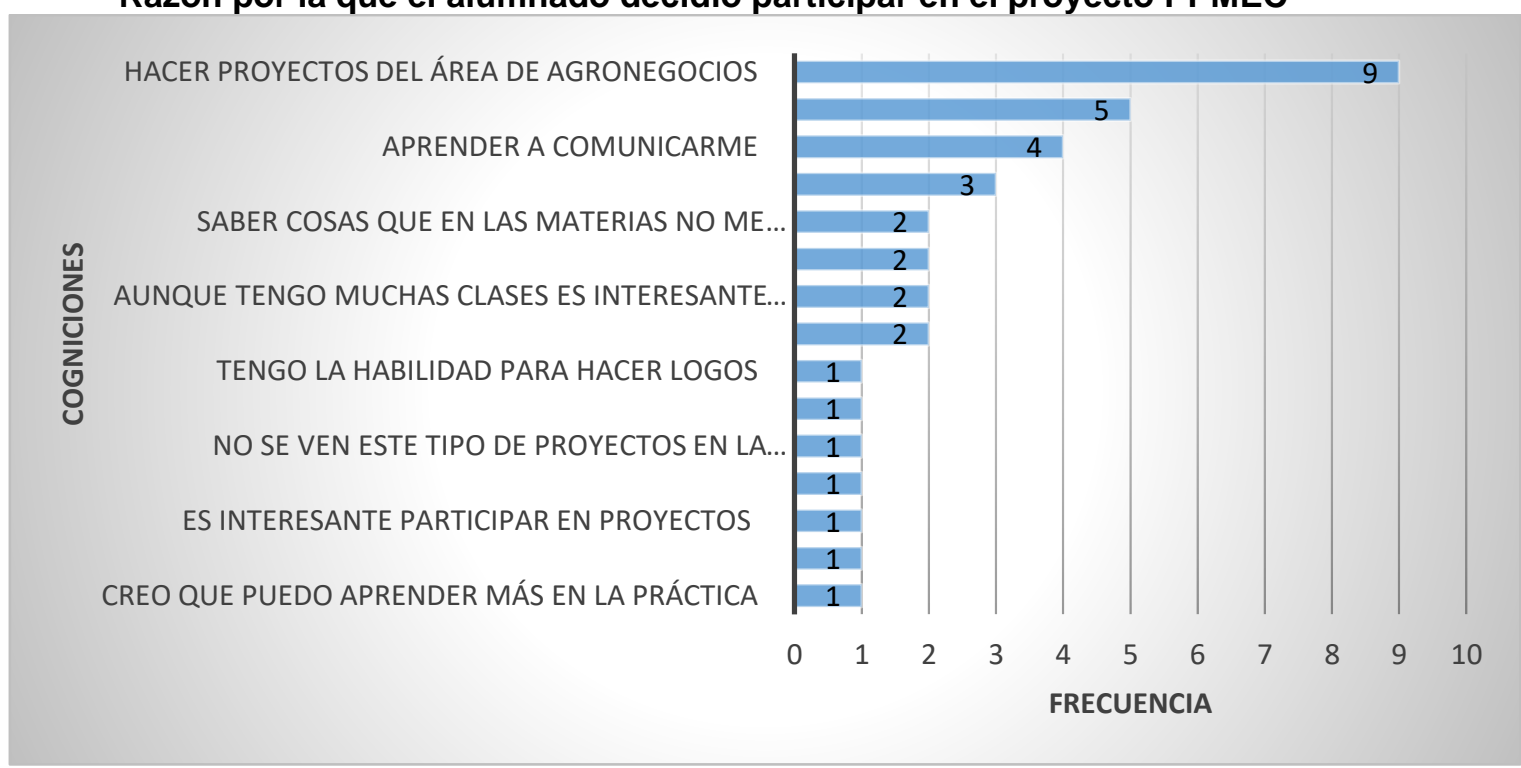

Fuente: Elaboración propia con la información generada de la aplicación de la encuesta inicial, 2015

La Figura 3 corresponde a la identificación de la idea principal que conciben el alumnado con respecto a su formación educativa, en la que predomina la descripción de los maestros con edad avanzada y ofreciendo materias tediosas y aburridas.

\section{Figura 3}

Concepción educativa que tiene el alumnado hacia su formación educativa

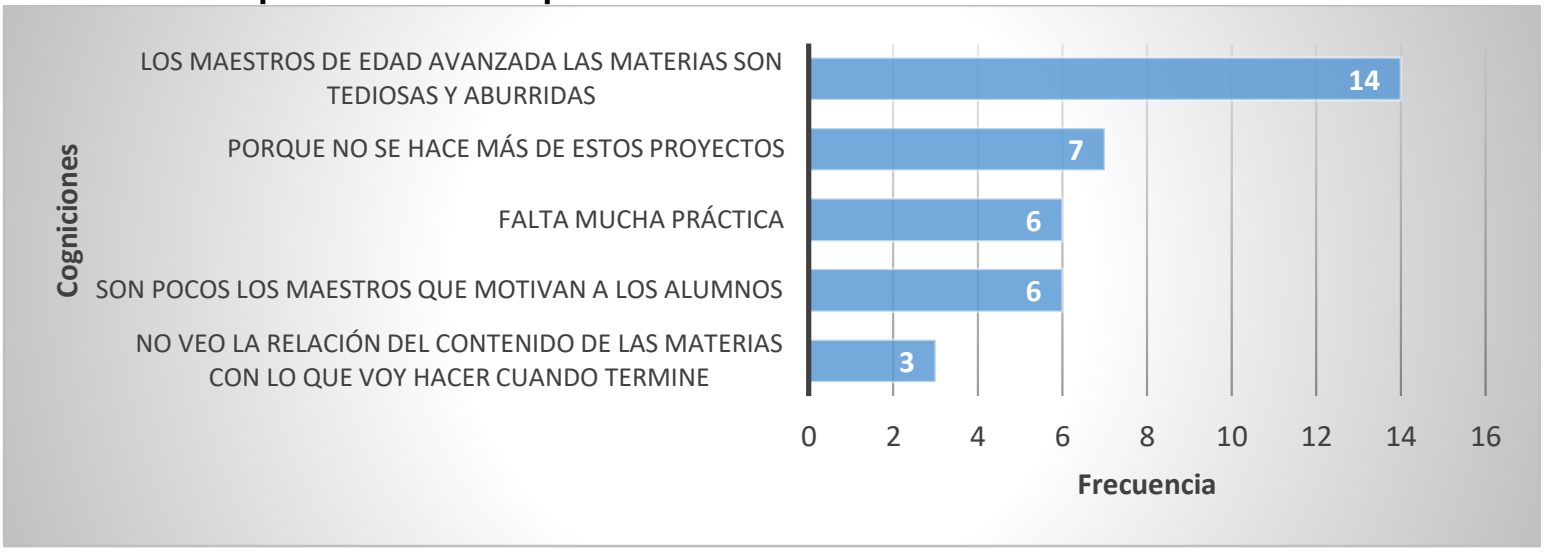

Fuente: Elaboración propia con la información generada de la aplicación de la encuesta inicial 2015 
Al inicio del proyecto los resultados de la entrevista realizada al alumnado que deseaban participar en el proyecto presentaron los siguientes resultados:

En el ámbito pedagógico, el alumnado no conoce el modelo educativo propuesto por la Universidad. El currículum formal no contempla materias en el área de comunicación, desarrollo humano y procesos productivos. Tal como identifica esta situación Trueba (2018, p. 10) señala que las verdaderas expectativas de desarrollo social y los objetivos y metas propuestas en los programas académicos "no solo no existe una verdadera correspondencia, sino que en numerosas ocasiones prevalece entre ambos una clara disyuntiva, o por lo menos una inocultable desvinculación".

El alumnado que participó en el proyecto manifiesta la inquietud de lograr identificar las actividades principales que desarrollará cuando sea profesionista, un indicador importante fue el miedo para hablar en público, son conscientes de la necesidad de que la institución establezca una incubadora de proyectos productivos, que es necesario tener mayor práctica, que les permita ver la relación del conocimiento con el posible ejercicio profesional, observan en los profesores y profesoras áreas de oportunidad en la didáctica, para motivar y hacer dinámico el proceso de enseñanza aprendizaje.

Con respecto a la concepción educativa general de su formación, no identifican algunos aspectos puntuales que pretende desarrollar el curriculum, les es difícil comprender la relación de los conocimientos que adquieren en el aula y la actividad que realizarán en la vida profesional.

Al iniciar la operación de PPMEC en el 2015 se tenía como objetivo principal atender y solventar las carencias que al alumnado había presentado y que se detectaron a partir de la revisión de fuentes documentales y la entrevista inicial; se pretendía establecer estrategias que a través de actividades complementarias al curriculum se reafirmara una formación Integral, reducir las principales debilidades detectadas en los egresados como son: pocos conocimientos prácticos, ser introvertidos, escaso sentido emprendedor, deficiente dominio del español, dominio del idioma inglés; se requiere que desarrollen la habilidad de comunicación, lograr que sean sociales ya que son reservados y sin facilidad de palabra.

\subsection{Nivel 2 Estratégico}

Para la puesta en marcha del programa se llevó a cabo la Planeación estrategia la cual se partió de establecer la misión y la visión del grupo. Correa (2005) concibe a la planeación como la articulación sistemática de las actividades que se requieren de un tiempo, espacio, 
información, técnicas, recursos y organización para su desarrollo. Es una especie de modelo experimental que busca orientar las actividades y aprovechamiento de los recursos y talentos, controlando al máximo el riesgo en el logro de los objetivos propuestos. Al momento de apropiarse el reto de la planificación hay que pronosticar tanto las condiciones internas como externas en los escenarios presentes y futuros.

A continuación se presenta la visión y la misión construidas para el desarrollo del proyecto (PPMEC).

Misión: Ser el grupo emprendedor identificado por su calidad de servicio, integración humana, y trabajo colaborativo, a través de desarrollar las habilidades necesarias para ser un profesional de calidad.

Visión Ser en el 2020 un equipo emprendedor consolidado, donde sus miembros (alumnado) lleven el liderazgo del proyecto integrando cada año nuevos participantes, para que los proyectos sigan vigentes en la institución. Las estrategias de aplicación parten de la publicación del proyecto para captar alumnado interesado, y detectar, a través de una entrevista, la inquietud por participar.

El diagnóstico sirvió de base para la planeación y la definición de los objetivos principales que son:

1. Contribuir a la formación integral de del alumnado a través de realizar actividades educativas, prácticas y de servicio a la sociedad mediante el desarrollo de proyectos productivos.

2. Conformar la primera incubadora de proyectos productivos multimedia en la Institución

3. Sentar las bases para lograr hacer realidad el Modelo Educativo con actividades complementarias al currículum,

4. Contribuir a la formación de del alumnado con habilidades comunicativas, el trabajo en equipo y los valores que conforman el ser.

5. Favorecer a la educación para toda la vida en la educación continua con procesos de capacitación.

6. Incidir en el uso de las TIC como herramienta didácticas y desarrollo de habilidades entre el alumnado.

Las Metas propuestas fueron:

1. Realizar por lo menos un Proyecto anual, con fines de concurso por recurso económico. 
2. Diseño e implementación de dos proyectos productivos anuales en el área de los agronegocios.

3. Ofrecer al alumnado que integran el proyecto un curso de capacitación semestral para el desarrollo de competencias duras y blandas.

4. Ofrecer los servicios de la puesta en marcha de proyectos productivos tanto en el sector público, privado y comunidad en general, elaboración y edición de videos confines de capacitación.

5. La asistencia a congresos para la difusión del proyecto (elaboración de ponencias) y proveer los materiales y capacitación necesarios para la organización.

En cada uno de los objetivos, así como en la misión y la visión se observa la esencia de vincular el conocimiento con la intervención social en beneficio de las comunidades tanto urbanas como rurales.

\subsection{Nivel 3 Organización.}

La naturaleza de organización durante el desarrollo de las actividades fue de trabajo en equipo y colaboración en la resolución de conflictos, la integración como la permanencia fue por libre decisión.

La democracia interna se manifiesta a través del proceso de organización, la coordinación es de un maestro líder del Programa. La integración se realiza a través de la convocatoria abierta a través de la cual se motiva a participar, los integrantes posteriormente se conforman en grupos según el interés de cada uno de ellos, en cada grupo se elige un líder. Actualmente el líder de PPMEC lo determinan los propios participantes con mayor antigüedad en el desarrollo de las actividades, ellos mismos informan del procedimiento, responsabilidad y compromiso que debe demostrar para lograr que PPMEC continúe en operación. El maestro coordinador para reuniones de trabajo solo tiene comunicación con el líder quien tiene la obligación de reunir el equipo a través de comunicación oral y de mensaje de textos. El proyecto es autofinanciable, de tal forma que se elige un responsable del recurso económico que se genere de las actividades realizadas, se promueve el desarrollo de capacidades de organización a través de cursos de capacitación.

En el año 2016 el alumnado participó en la puesta en marcha de proyectos de intervención social a través del desarrollo del curso de educación continua. 
Al desarrollar estas actividades el alumnado desarrollaron las habilidades de trabajo en equipo, resolución de problemas y realización de videos de promoción y captación de donativos de materiales.

Uno de estos programas de educación continua es de "Elaboración de Productos Lácteos" donde el alumnado realizó actividades desde trabajo de limpieza y mantenimiento en establos lecheros y granja porcina y la introducción al siguiente cuso de Industrialización de cárnicos.

Otro proyecto fue el Desarrollo de Huertos escolares, el equipo PPMEC inicio sus actividades en las estancias infantiles del perteneciente a la dependencia del ( DIF) Desarrollo Integral de la Familia del municipio de Saltillo Coahuila México donde ofrecieron capacitación a niñas y niños de edades para lograr la producción de hortalizas, aprovechar el desecho orgánico, generar sustrato para mejorar las condiciones de la tierra, se determinó la ventaja de orientar en la elaboración de composta; este proceso fue el primero en el que el alumnado participó en la producción de un producto multimedia, fue una experiencia satisfactoria ya que el alumnado de la universidad tuvieron un acercamiento con niños en edad maternal y preescolar: este tipo de vinculación es difícil encontrarla, es decir, establecer colaboración entre la etapa inicial y la etapa final de la educación, las emociones, sentimientos, el cuidado en la atención que se brindada a los menores se manifiesto con la alegría o gusto por el servicio, la enseñanza de lograr producir y cosechar su propio alimento, la capacitación para escuela para padres que se enfocaba en lograr concientizar sobre el desarrollo de cultivos de huertos ya sea en traspatio y en el cultivo sin patio.

Este proyecto fue presentado en el Congreso Internacional de la AMECYD Asociación Mexicana de Educación Continua y a Distancia, con sede en la Universidad Autónoma de Nuevo León, del 19 al 21 de octubre de 2016 El resultado principal obtenido fue demostrar que se puede lograr la vinculación entre el nivel superior de educación y el inicial es decir las estancias infantiles, al promover la formación integral.

La idea de PPMEC al promover la elaboración de productos multimedia es facilitar la comprensión de los proyectos de educación continua de la UAAAN. Aunque parece sencillo y probablemente por algunos expertos, como algo insignificante, la experiencia en estos proyectos fue muy importante, porque el alumnado desarrolla otras habilidades y los ponentes del congreso los reconocieron y apreciaron su labor complementaria a su formación y les dijeron que estos trabajos son la base del desarrollo de muchas actividades que se emprenden en las Instituciones de Educación Superior. 
La experiencia de proyectos multimedia de educación continua se presentó en la ciudad de México como un proyecto de busca de la sustentabilidad del municipio de Saltillo Coahuila, ante la Asociación Nacional de Alcaldes (ANAC) el 11 de diciembre 2016 obteniendo el reconocimiento por el trabajo realizado en las estancias infantiles.

A partir del año 2017 derivado de la participación en el congreso y el premio logrado por el Municipio de Saltillo, el grupo PPMEC fue reconocido por la Dirección General Académica de la UAAAN como un grupo emprendedor registrado institucionalmente.

A la fecha, se continúa trabajando en las estancias infantiles del DIF Saltillo, el alumnado es quien genera la campaña para su continuidad. Entre las actividades realizadas se encuentra ser acompañantes del profesorado para desarrollar cursos de Educación Continua, entre los que se encuentran: Polinización del Maíz, Industrialización del Nopal con tres videos: Cristalizado de Nopal, Nieve de Nopal y Licor de Nopal y Poda del manzano. Durante este tiempo también se desarrolló la tesis nivel licenciatura de Diana Laura Marín Lara; trabajo de observación, estudio y obtención de información de las hierbas y arbustos que crecen de manera natural en el campo de las comunidades del sureste del municipio de Saltillo; Entre las que se encuentran el tomillo, mejorana, laurel, rosa de castilla, sábila, sangre de grado, y gobernadora, las cuales han aprovechado para diversos usos como remedios caseros, condimentos, o para elaborar productos de limpieza.

Otro proyecto que surge de la inquietud de apoyar la economía familiar, se ha desarrollado como opción de generación del agronegocio en el área silvoagropecuario es un horno de deshidratación que se encuentra en fase de diseño.

Durante el 2019 surgió la oportunidad de trabajar de manera interdisciplinaria colaborativa con la Universidad Autónoma de Coahuila particularmente con la Facultad de Ciencias de la Comunicación, en el proyecto: "Comunidades emergentes de conocimiento y procesos de Investigación/ creación audiovisual" (CEC-PICA) financiado por el CONACYT en la modalidad de Investigación Científica Básica, cuyo objetivo es "desarrollar una reflexión teórica y metodológica sobre la generación del conocimiento científico en entornos multiculturales, multidisciplinares y multigeneracionales, que permita diseñar estrategias para la construcción colectiva y la gestión de dicho conocimiento en diversos formatos y para comunidades no académicas” (Moreno, 2019, p. 1). 


\subsection{Nivel 4 Multimedia}

Experiencia de utilizar las TIC para el desarrollo de habilidades de comunicación oral y escrita, los resultados al respecto se correlacionaron dos variables la participación en el desarrollo de los proyectos productivos de Educación Continua y las habilidades adquiridas por el alumnado, se logró verificar que el alumnado participante, en su totalidad mejoraron sus habilidades de comunicación oral y escrita, al participar en la preparación de guiones de grabación, secuencia de contenidos multimedia, y de igual forma la comunicación oral ya que perdieron el miedo de hablar en público, estructuraron habilidades del pensamiento para su dialogo verbal frente a cámaras al realizar los videos.

Resultados concretos con las actividades multimedia, desarrollaron el valor de la responsabilidad, ética, compromiso, la actitud de servicio, el trabajo en equipo y la resolución de conflictos al comprometerse en llegar a buen término cada uno de los proyectos que emprendieron. Para la institución colaboraron a lo largo de este tiempo en la preparación cinco cursos multimedia los cuales se encuentran en funcionamiento ofreciéndolos a los productores y público en general a través de la plataforma institucional. Entre los cuales se encuentran: Poda del manzano, Prueba de pluviometría con pivote central, Industrialización del nopal el cual contiene tres videos, nieve de nopal, licor de nopal y cristalizado de nopal, elaboración de composta, elaboración de lombricoposta, todos estos cursos tienen como objetivo apoyar a la capacitación para el desarrollo de proyectos productivos en el área de los agronegocios. El considerar desarrollar proyectos multimedia ligados a los agronegocios fue el elemento clave para el desarrollo de los proyectos, a través de ellos se logra formar las competencias duras y blandas.

Los aspectos generales que se pueden utilizar para el complemento curricular son los siguientes: La planeación puesta en marcha, desarrollo y evaluación de proyectos tanto multimedia como de cualquier otro tipo a través de los cuales el alumnado es capaz de complementar su formación profesional, al poner en práctica el aprender a aprender, el aprender a hacer y el aprender a ser, se hace realidad una formación integral, proporcionando al alumnado una perspectiva mayor en su vida profesional.

\subsection{Nivel 5 Intervención Social}

Se detecta de manera trasversal en cada una de las actividades desarrolladas.

Como resultado de la intervención social del desarrollo de proyectos se presentan los resultados enfocados al desarrollo de formación integral considerando las competencias duras 
y blandas, logrados en el alumnado a lo largo de estos 5 años y aquellas características que describen a 12 participante que se mantuvieron líderes desde el inicio del programa hasta su graduación en el 2019

Una variable significativa fue el que al trabajar en equipo y por pertenecer a carreras diferentes unos aprendieron de otros, se identifica los contenidos que vinculan la promoción del aprendizaje significativo a través de fomentar el saber aprender, saber hacer y saber ser. En la Figura 4 se puede revisar las competencias duras y blandas que se desarrollaron a través del proyecto como completo al curriculum.

Figura 4

Desarrollo de competencias como complemento al currículum

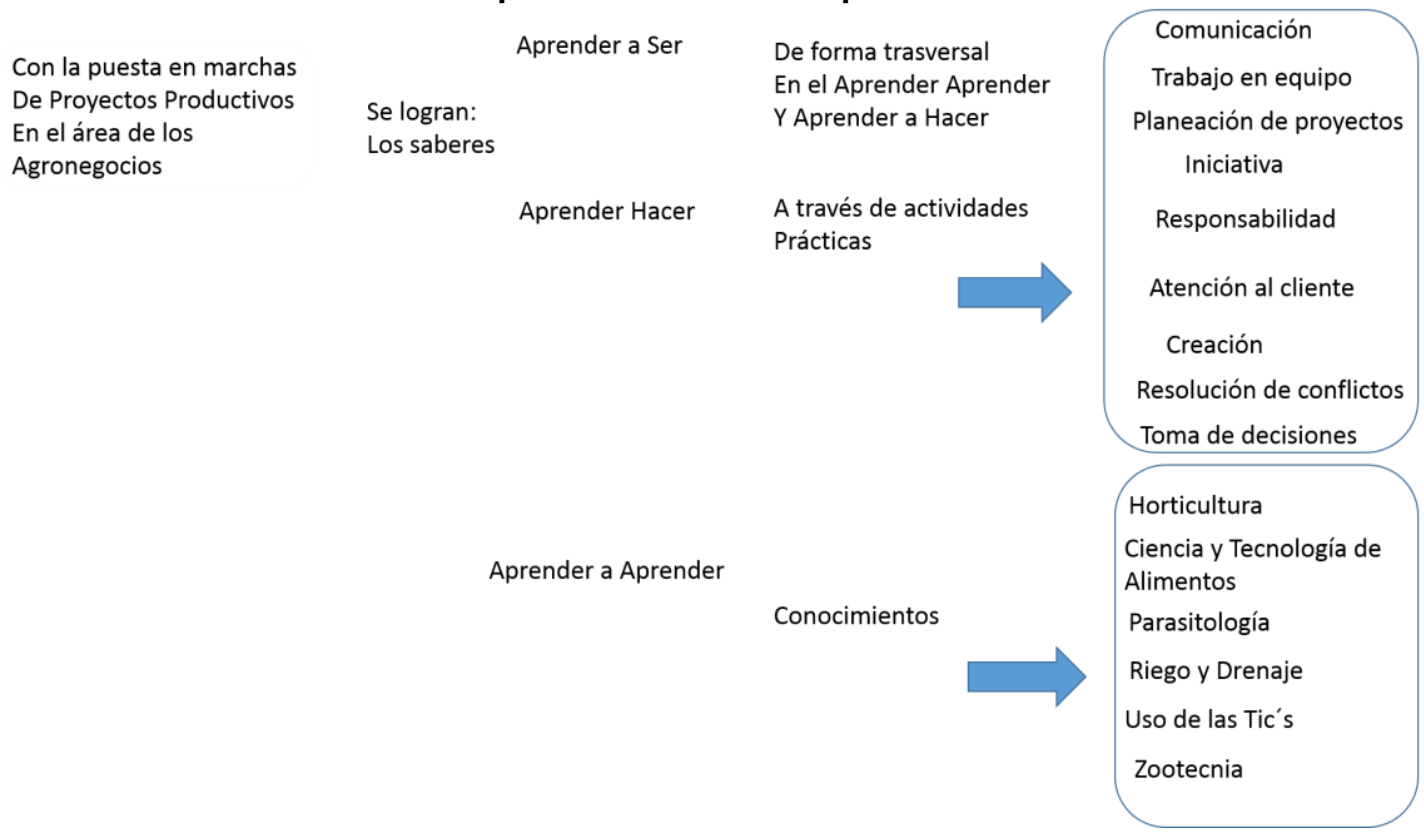

Fuente: Elaboración propia, 2020, a partir de los resultados de la investigación

Se describen a continuación las características del alumnado participante un total de 30 que iniciaron sus actividades en el proyecto en el año 2015, así como el alumnado que se mantuvieron líderes del proyecto los cuales se son 12 hasta su graduación en el 2019.

En la Figura 5, se puede observar las características básicas con las que contaban el alumnado, como el que su segunda lengua es el español después de una lengua indígena y con la formación recibida a través del proyecto, lograron desarrollar habilidades de comunicación, trabajo en equipo y realizar prácticas y movilidad académica en el extranjero. 
Figura 5

Características de los estudiantes PPMEC

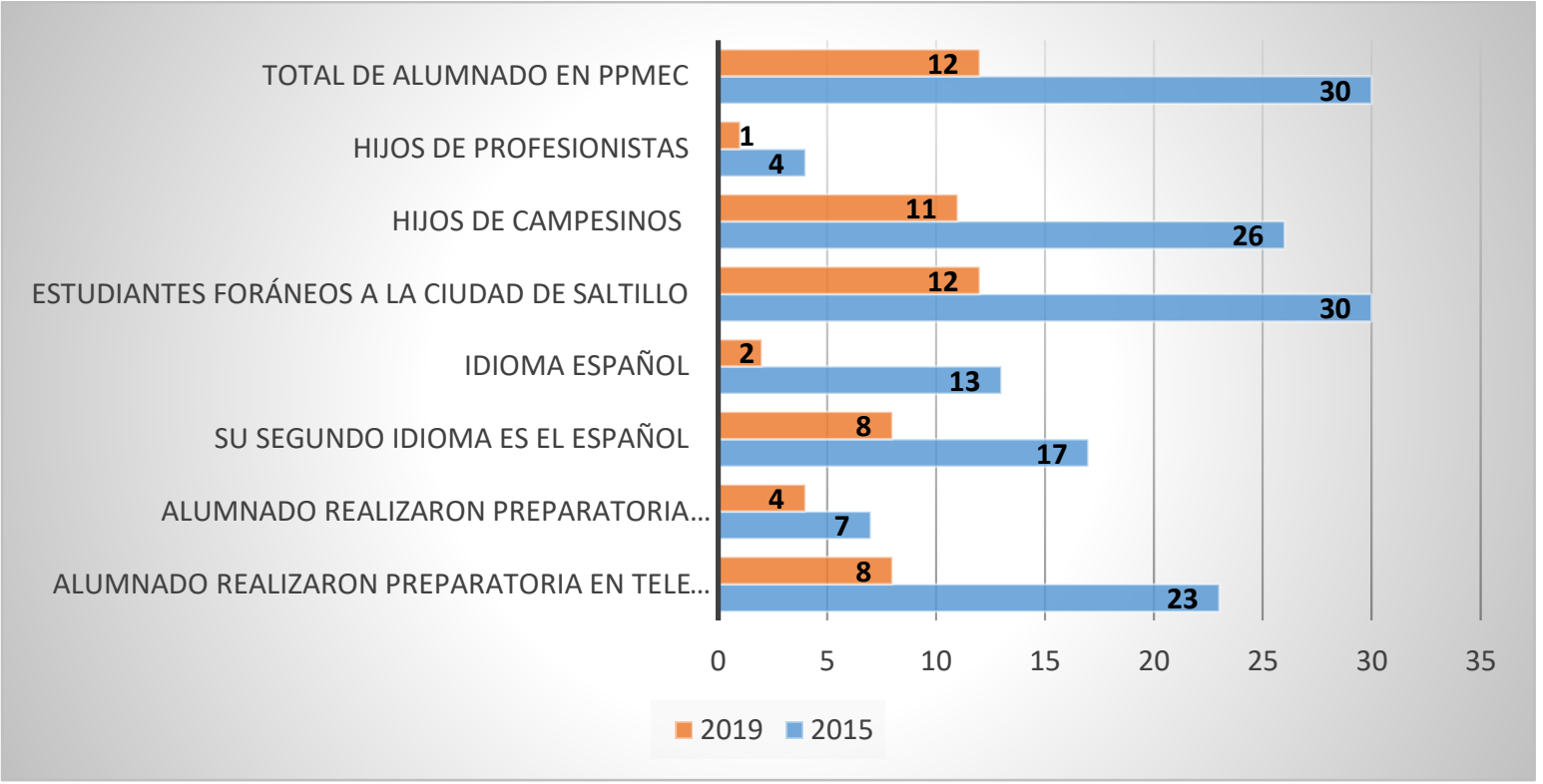

Fuente: Elaboración propia con la información generada de la aplicación de la encuesta inicial 2015

En la Tabla 1 se describe las habilidades, destrezas y valores que el alumnado PPMEC logró desarrollar durante su estancia en el programa, básicamente en la participación en cada una de las actividades planeadas, se identifican competencias blandas que se requieren actualmente para el desarrollo profesional. Esta información se genera a partir de la guía de observación que los profesores y profesoras colaboradores en el programa realizaron para la presente investigación.

Tabla 1

Habilidades, Destrezas y Valores obsevados en el alumnado PPMEC

\begin{tabular}{|l|l|l|}
\hline Habilidades & Destrezas & Valores \\
\hline Autoconocimiento. & Liderazgo & Honestidad \\
\hline Empatía. & Organización & Sensibilidad \\
\hline Comunicación asertiva. & Capacidad resolutiva & Gratitud \\
\hline Relaciones interpersonales. & $\begin{array}{l}\text { Habilidades para trabajar bajo } \\
\text { presión }\end{array}$ & Humildad \\
\hline Toma de decisiones. & Manejo de cámaras fotográficas & Prudencia \\
\hline Solución de problemas y conflictos. & Mejo de mecanismos de audio & Respeto \\
\hline Pensamiento creativo. & Logística de entrada & Responsabilidad \\
\hline Pensamiento crítico. & Logística de salida & \\
\hline Comunicación oral y escrita & & \\
\hline
\end{tabular}

Fuente: Elaboración propia con la información de las guias de observación realizadas para la presente investigación en un periodo del 2015 al 2019. 
Se logró identificar que el alumnado que se mantubo lideres del proyecto (12) además de desarrollar habilidades y valores, desarrollaron las siguientes destrezas: Liderazgo, organización, capacidad resolutiva, habilidad para trabajar bajo presión, lo cual les permitió realizar movilidad internacional, prácticas profesionales internacionales, titularse inmediatamente al terminar sus creditos y contar actualmente con un trabajo estable.

\section{Conclusiones}

¿Se puede desarrollar competencias en el alumnado con actividades complementarias al currículum?

Es factible desarrollar competencias duras y blandas en el alumnado sin la necesidad de que estén manifestadas en un modelo educativo o en un currículum formal. La puesta en marcha de este tipo de proyectos que pretenden desarrollar alguna actividad productiva, permite reunir el aprender con el aprender a hacer, y promueve el aprender a ser en las personas participantes. La esencia radica en el quehacer educativo que establece como sostén la disposición por parte del profesorado con la participación del alumnado motivado por una actividad, esto provoca el desarrollo de las competencias.

El profesorado al realizar su actividad ha de determinar la educación como un factor de quehacer social explícito, correlacionado tanto con las condiciones, culturales y políticas, se puede definir con certeza que la voluntad de los dos factores del proceso de enseñanza aprendizaje hace la diferencia en la puesta en marcha de cualquier tipo de proyecto, y en esto radica el éxito logrado en el alumnado que participó en el proyecto, ya que se dedicaron a construir el significado.

La experiencia aquí plasmada, se ha logrado a partir de la idea de construir un mismo proyecto en el que la creatividad y la resolución de conflictos imperan en todo momento; además del acompañamiento, para que el alumnado logre un aprendizaje significativo. Es gratificante contribuir a un proceso de formación integral.

El horizonte teórico revisado sobre competencias proporciona los fundamentos en los que, quienes participan en la formulación de alternativas para el desarrollo de competencias, pueden identificar la importancia del desarrollo de estas en el alumnado, pero se habla de un sentido ideal; es difícil encontrar procesos educativos reales y significativos de formación integral en los cuales se haga realidad la teoría, hay que aprender la lección que nos ha dejado el tiempo en área de competencias, es decir actualmente es el momento en que el profesorado debe planear para actuar, promover para lograr y evaluar para crear. 
El conjunto de investigadores que participaron se encuentran conscientes del potencial que proporciona el modelo de intervención educativa a través de la planeación y puesta en marcha de un proyecto productivo. El cambio de la práctica educativa hacia la esencia del desarrollo de competencias, estén o no declaradas en el currículo, permite promover la formación integral del alumnado.

El desarrollo de proyectos productivos o de cualquier otro tipo con fines educativos es un mecanismo claro para lograr la vinculación universitaria y establecer el compromiso social con la comunidad.

Se considera que la problemática en este tipo de proyectos es la continuidad Institucional. El alumnado participante requiere del apoyo y no se habla del apoyo económico, sino del respaldo institucional. Es decir, el pretender lograr la educación integral del alumnado a través de la puesta en marcha de proyectos se ha de establecer desde la determinación de propuestas de la base magisterial, considerar ser sistemáticas, es decir lógicas, coherentes una con la otra, los objetivos estarán basados en el análisis de la realidad y en los medios al alcance, tanto humanos como materiales; Las actividades deberán estar en concordancia con la estrategia general, el objetivo a seguir y las tareas estructuradas, de tal forma que una permita desarrollar otra.

Este tema queda abierto a nuevas reflexiones, pero es indispensable que el desarrollo de las competencias haga posible la apertura de todo tipo de alternativas de formación integral.

\section{Referencias}

Castro, Jesús. (2018). La Narro, 95 años de dar vida al campo mexicano. Periódico Zócalo, Saltillo, Coahuila. México. Recuperado de https://www.zocalo.com.mx/new site/articulo/la-narro-95-anos-de-dar-vida-al-campomexicano

Correa, Cecilia. (2005). Administración estratégica y calidad integral de las Instituciones Educativas. Bogotá, D.C. Colombia: Cooperativa editorial Magisterio.

Delors, Jaques. (1994). Los cuatro pilares de la educación. En La Educación encierra un tesoro (pp. 91-103). Madrid, España: UNESCO.

Habermas, Jurgen. (1987). La teoría de la acción comunicativa (Vols. 1 y 2). Madrid: Taurus.

Maldonado, Miguel. (2010). Currículum con enfoque de competencias. Bogotá: Ecoe Ediciones.

Monereo, Carles., y Pozo, Juan. (1999) El aprendizaje estratégico: enseñar a aprender desde el curriculum. España: Santillana. 
Moreno, Adriana. (2019). Comunidades emergentes de conocimiento y procesos de Investigación/ creación audiovisual. Saltillo, Coahuila: (CEC-PICA) Universidad Autónoma de Coahuila.

Morín, Edgar. (2000). Los siete saberes necesarios para la educación del futuro. Bogotá: Ministerio de Educación Nacional.

Müller, Ricardo. (2010). Manual para la presentación de proyectos productivos sostenibles. Lima, Perú: FONDOEMPLEO.

Ortiz, Fidel. (2018). Modelo de incubadora para generar emprendimientos con alto valor agregado. Estudios del emprendimiento contemporáneo en América Latina. Bolivia: ECORFAN.

Senge, Peter. (1993) La quinta disciplina: Cómo impulsar el aprendizaje en la organización inteligente. Buenos Aires: Granica SA-Adelphi S.A.

Singer Marcos., Guzmán, Ricardo., y Donoso, Patricio. (1999). Entrenando Competencias Blandas en Jóvenes. Recuperado de: http://www.inacap.cl/tportal/portales/tp90b5f9d07o144/uploadlmg/File/PDF/Entrenand o Competencias Blandas en Jovenes.pdf

Raynaudo, Gabriela., y Peralta, Olga. (2017). Cambio conceptual: una mirada desde las teorías de Piaget y Vygotsky. Liberabit, 23(1), 110-122. Recuperado de http://dx.doi.org/https://doi.org/10.24265/liberabit.2017.v23n1.10

Tobón, Sergio. (2006). Competencias en la educación superior: Política s hacia la calidad. Bogotá: Ecoe.

Trueba José. (2018). Los modelos educativos en México Una perspectiva Históricasociológica. Guanajuato, México: Universidad de Guanajuato.

Universidad Autónoma Agraria Antonio Narro (UAAAN). (1995). Modelo Educativo. Saltillo Coahuila, México: Editorial UAAAN.

Universidad Autónoma Agraria Antonio Narro (UAAAN). (2012). Estudio de pertinencia de los programas educativos. Saltillo Coahuila, México: Editorial UAAAN. 
Revista indizada en

scip/o redalyc sy latindex

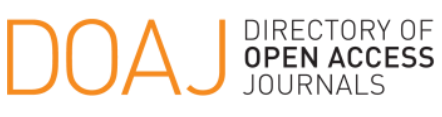

Distribuida en las bases de datos:
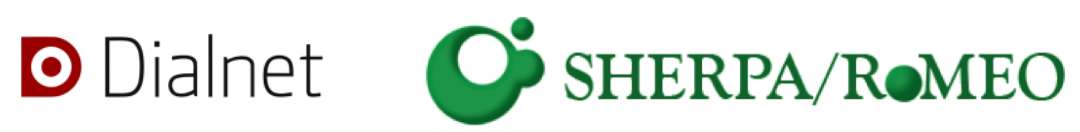

REDIB

Red Iberoamericana

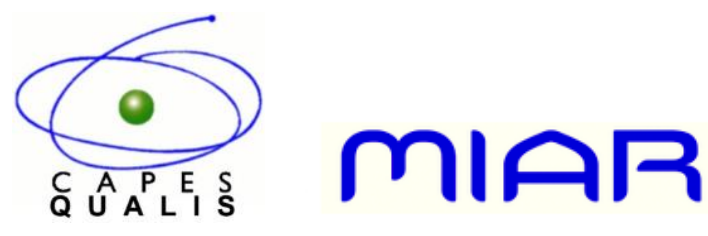

